\title{
An Underactuated Propeller for Attitude Control in Micro Air Vehicles
}

\author{
James Paulos and Mark Yim
}

\begin{abstract}
Traditional coaxial helicopter micro air vehicles use a large propeller motor in conjunction with two small servomotors to control thrust, pitch, and roll forces and moments. Quadrotors similarly generate these necessary forces and moments through the coordinated control of multiple actuators. We present a novel propeller architecture which allows a single motor and rotor to express such control by modulating the torque applied to one passively hinged, underactuated propeller. Flight tests of a two-motor coaxial helicopter demonstrate that such a system can provide active stability and control in a real flight system.
\end{abstract}

\section{INTRODUCTION}

Large scale unmanned air vehicles (UAV) have provided an eye in the sky to support a variety of applications. Imagining future roles for UAVs in cluttered urban settings or indoor environments has driven efforts towards palm-sized micro air vehicles (MAV). The small size of MAVs can permit them to be uniquely agile [1]. In addition to being able to physically access confined spaces, their low inertia further allows the possibility of safe, recoverable collisions with structures and people [2]. Hover-capable MAVs are of particular interest for indoor applications, with examples including quadrotors [3], coaxial helicopters [4], [5], and ornithopers [6], [7].

The present miniaturization of MAVs owes primarily to advances in improved battery power densities, better use of light composite materials, shrinking electrical components and MEMS sensors. A principle difficulty lies in the fact that as these flight systems have grown smaller, their complexity has remained largely unchanged. A one hundred gram quadrotor or coaxial helicopter is nearly a component-forcomponent replica of its one kilogram or even ten kilogram cousin, at a smaller scale. This direct miniaturization has proven easier for some architectures, with the simple motor and fixed pitch propeller of quadrotors being more cooperative than the servo-driven swashplate mechanisms of common coaxial helicopters.

Most contemporary MAVs capable of hover and vertical flight employ similar lifting rotors for thrust but differentiate themselves through the way in which attitude control moments are achieved. Traditional helicopter design has been informed by the assumption that large thrust actuators have slow characteristic response, necessitating separate actuators and a complex linkage system for high bandwidth attitude control. In contrast, four-rotor "quadrotor" flight systems have shown that this strict dichotomy need not hold, and these devices instead coordinate multiple identical rotors to achieve both thrust and moment objectives. Finally, some efforts have been made to achieve both thrust and moment response from a single actuator using indirect drive through

The authors are with the Dept. of Mech. Eng. \& Appl. Mechanics and GRASP Lab at the University of Pennsylvania, Philadelphia, PA. jpauloseseas. upenn. edu, yimegrasp.upenn.edu a linkage [8]. This paper presents a new concept a MAV propulsion system capable of using a minimum number of actuators in dual rolls. This simplifies and lowers the cost of MAVs. Removing complex swash plates and reducing the number of actuators reduces the number of parts, thus increases reliability (fewer parts to fail), reduces maintenance costs, reduces vehicle mass, and reduces manufacturing costs. Experimental results for the actuator response are presented along with a demonstration of a full flight vehicle using this system for both active stability and maneuvering.

\section{Control Concept}

Traditional small scale helicopters and coaxial helicopters already use a single rotor to generate both thrust force and attitude moments. Broadly speaking, thrust comes from the average speed and angle of attack of the propeller blade, and attitude moments are derived from an added cyclic oscillation in the angle of attack through each revolution. This cyclic pitching blade motion is ordinarily proscribed by a swashplate linkage driven by two or three additional servomotors and is sometimes augmented by the dynamics of a stabilizing flybar [9]. In contrast, we can attain this motion directly from a passive dynamic response of the propeller itself. For such a propeller the mean applied torque sets the rotational speed and thrust, and an applied oscillatory torque induces the desired cyclic oscillation in blade pitch.

A simple realization for such an underactuated propeller consists of a rigid hub linked to two semi-rigid airfoil blades, as illustrated in Figs. 1-2. The vertical motor shaft is fixed to the central hub and transmits torque to the rotor hub. Propeller blades are attached to this hub on either side through simple hinges. The axes of the two hinges lie in the same plane as the vertical propeller axis of rotation, but they are not parallel to the axis of rotation as would be found in typical helicopter rotors. The blade denoted the 'positive' blade has the top of its hinge inclined towards the central shaft. Conceptually, this 'positive' rotor responds to an impulsive torque on the hub by flexing backwards and exposing increased blade pitch as shown in Fig. 3. Similarly, a retrograde torque causes the 'positive' rotor to flex forwards on its hinge and decrease its pitch. The opposing 'negative' blade has the top of its hinge axis inclined away from the central shaft, and the complementary geometry creates an opposite response to torques. By superimposing a sinusoidal torque at the rotor frequency on top of the steady torque needed to balance rotor drag, a cyclic oscillation in blade pitch is induced that is phase locked with the rotor position.

The motion is functionally similar to that proscribed by a swashplate mechanism, but it is now produced through actuation of only the single main motor. This motor can now generate attitude moments by, for example, selectively elevating blade pitch in the northern sector and decreasing it in the southern sector through each revolution. By changing 


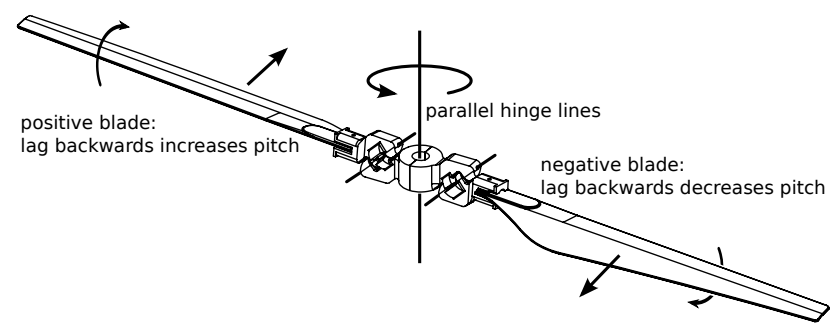

Fig. 1. Two passive hinges allow a cyclic applied torque to drive a cyclic pitch oscillation.

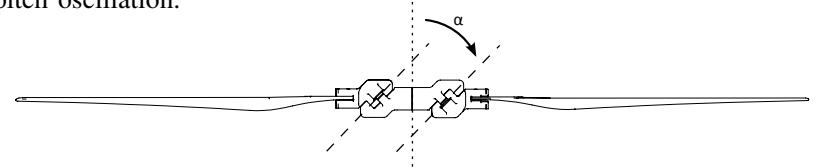

Fig. 2. Hinge lines are in the plane of the rotor shaft, but inclined from vertical by an angle $\alpha$.

the magnitude of the driving sinusoidal modulation the magnitude of the control moment is adjusted, and by changing the phase of the signal relative to the airframe the direction of the control moment in the pitch and roll plane is affected. This linkage can be a simple modification to a propeller blade by manufacturing a flexure joint into an injection molded plastic blade. Forming the blade from a low cost material like polypropylene with good fatigue properties would allow it to withstand many repeated cycles of small angle bending.

A complete vehicle may gain authority over yaw by pairing this novel rotor with a traditional helicopter tail rotor or by adopting a coaxial rotor configuration. In either case, the resulting two actuator system achieves the same degree of control freedom as either a traditional four actuator helicopter or four motor quadrotor. The comparative philosophy of this approach is that the complexity and cost is removed from the physical device and shifted to the control. We can then exploit the advances in computational power, reduced size, power consumption, and cost for these electronic systems.

\section{IMPLEMENTATION}

A physical realization of the proposed propeller system is shown in Fig. 4. The drive motor is a Park 400 12-pole, $740 \mathrm{Kv}$ brushless motor suitable for direct drive of large propellers. The three part propeller hub was manufactured on an Object 3D printer from a plastic polymer. The fixed hub interfaces with the motor shaft using a commercial aluminum mandrel, and two custom plastic blade clamps connect to the central hub along steel wire hinges. The aerodynamic elements are commercial propeller blades from E-flite modified to interface with the custom rotor clamps. The resulting propeller has a rotor disk diameter of $38.6 \mathrm{~cm}$ and generates approximately $1 \mathrm{~N}$ thrust at $1200 \mathrm{rpm}$.
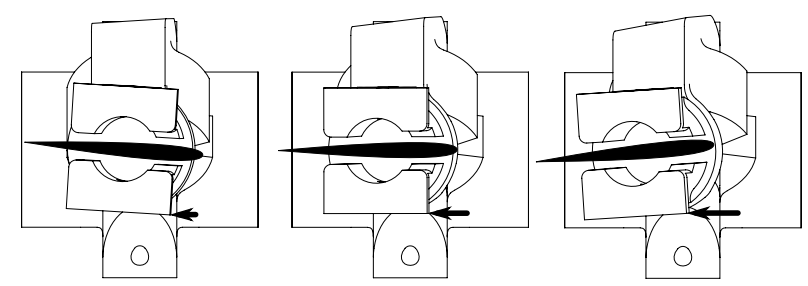

Fig. 3. As the positive blade lags backwards, the pitch increases.

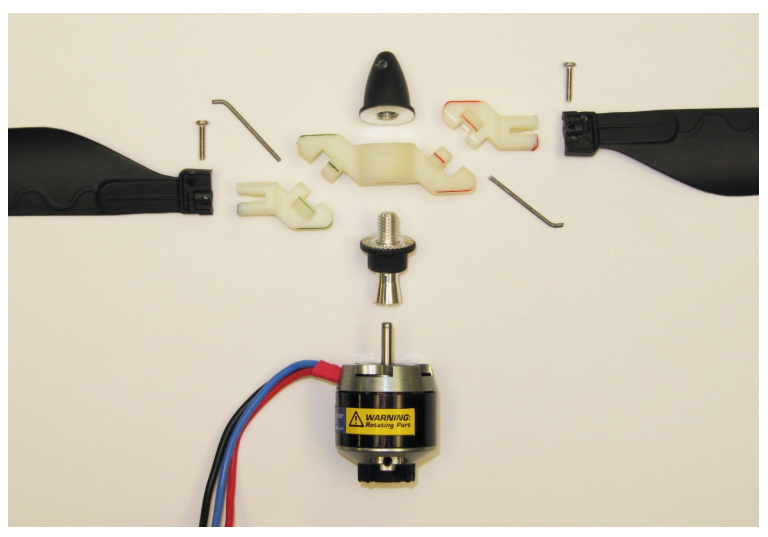

Fig. 4. Hinged propeller components.

TABLE I

COMPONENT MASSES OF THE PROPELLER AND Motor

\begin{tabular}{|r|l|}
\hline component & mass \\
\hline motor & $54.8 \mathrm{~g}$ \\
\hline mandrel & $6.9 \mathrm{~g}$ \\
\hline hub & $3.7 \mathrm{~g}$ \\
\hline two blade clamps & $1.6 \mathrm{~g}$ \\
\hline two airfoils & $8.8 \mathrm{~g}$ \\
\hline \hline total & $75.8 \mathrm{~g}$ \\
\hline
\end{tabular}

Cyclic control of the motor torque requires knowledge of the absolute rotor position - information not available from off the shelf brushless motor controllers designed for flight systems. Custom computation inside the inner control loop is also required to synthesize the appropriate high frequency modulation from an input amplitude and phase offset. A custom high performance brushless motor controller was developed which fulfills both requirements while also providing bidirectional communication and diagnostic capabilities useful for evaluation. Use of this controller requires mounting an external magnetic encoder which resolves the rotor position to $1 / 4096$ of a revolution. The controller can drive $10 \mathrm{~A}$ continuously from common $7.4 \mathrm{~V}$ or $11.1 \mathrm{~V}$ lithium polymer batteries. The effective applied motor voltage at any instant is adjusted through a $50 \mathrm{kHz}$ pulse width modulation (pwm) signal with 300 steps of resolution.

\section{Force And Moment Measurements}

A fixed test stand was used to measure the open loop propeller response in a controlled environment. A custom bracket holds the motor and constrains the rotor position sensor. The propeller assembly of Fig. 4 attaches to the output shaft of the motor. The motor bracket mounts to a six-axis force and torque sensor atop a rigid pylon as shown in Fig. 5. The rotor is placed 1.25 diameters above the nearest flow obstruction in order to avoid ground effects which might artificially inflate measured thrust performance.

An ATI Nano 17 force/torque sensor sends analog signals to a 12-bit NI DAQ sampling at $20 \mathrm{kHz}$. A MATLAB code interprets the six strain gauge channels to calculate the forces in three directions and moments about three axes imposed on the face of the sensor. This system resolves $1 / 320 \mathrm{~N}$ of force and 1/64 N.mm torque, and characteristic magnitudes in this application are thrust forces of approximately $1 \mathrm{~N}$ and moments of tens of $\mathrm{N} \cdot \mathrm{mm}$. The test script also captures the motor controller's own measurements of rotor position, speed, and applied pwm input at a sampling rate of $500 \mathrm{~Hz}$. 


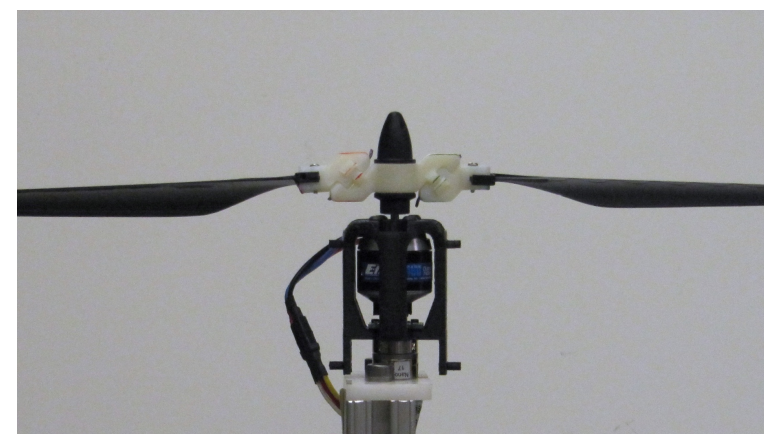

Fig. 5. The black plastic motor bracket was mounted to a small load cell and supported out of ground effect on an aluminum pylon.

Experiments evaluating rotor lift and moment capabilities were conducted from a power supply at $7.4 \mathrm{~V}$, but the eventual flight vehicle was powered by a lithium polymer battery at $11.1 \mathrm{~V}$ in order to increase excess thrust margins.

\section{Photographic Methods}

An entire cycle of the driven pitch variation completes for every revolution of the propeller. With normal operating speeds in excess of $1200 \mathrm{rpm}$, the response shape displays in less than $1 / 20$ of a second. The control output performance of the propeller system can be evaluated through the force and moment sensor data which easily resolves this behavior at a sample rate of $20 \mathrm{kHz}$. However, direct observation of this high speed motion is critical to confirming what mechanisms are actually at work.

A strobe-based low cost photography system was developed to enable accurate visualization. While even fast shutter speeds on the order of milliseconds record significant motion blur, the flash of a xenon strobe can have a duration shorter than 1/13,000 of a second. By taking a long exposure photograph of the propeller in motion in a darkened room and firing the strobe at the desired moment, crisp images of the spinning propeller can be captured such as those of Fig. 6. Since the cyclic behavior is identical on every revolution and is phase locked with the rotation angle of the motor, it is possible to capture a sequence of images with the rotor in different positions and interpret them as a high resolution, high speed video. A microcontroller automates this process by opening the camera shutter, waiting for the rotor to reach a reference position, and then flashing the strobe after a precise, adjustable delay. A MATLAB script sequences many photographs with increasing delay times to capture a complete revolution of the propeller. The resulting synthetic video illustrates the blade motion with 3000 x 4000 pixel frames captured at 1/1000 second intervals. Similarly, photographs may be captured with the rotor in one polar position but with varying torque modulation phase, and the resulting stack of photographs illustrates the propeller's oscillatory motion as viewed by an observer rotating with the rotor. Such photographs reveal the minimum, trim, and maximum pitch angles shown in Fig. 6. The success of these photographic methods confirms that the blade oscillations are both identical at each repetition and phase locked to the rotation of the motor. The video clearly resolves the motion about the hinge line as well as elastic bending of the propeller blade due to the varying thrust at different polar angles around the rotor disk.

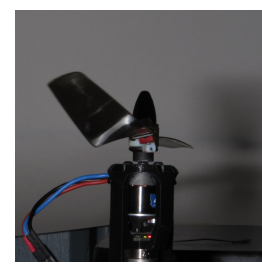

(a) high pitch

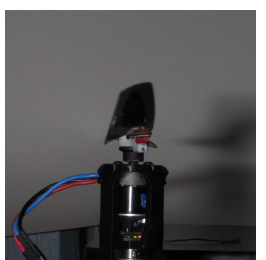

(b) trim

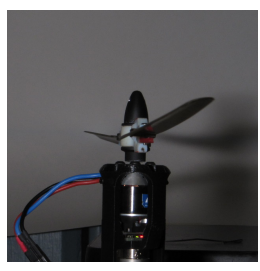

(c) low pitch
Fig. 6. The instantaneous pitch at one location is controlled by the phase of the torque modulation. Strobe photos are captured at operating speed.

\section{Measured Control Moments}

The most important property of the rotor system as an attitude actuator is the mapping of input signal to generated moment. To characterize this, the rotor was driven to a steady nominal operating condition and then oscillatory control was applied across a range of drive amplitudes on the test stand. Data from the six-axis force and torque sensor was used to calculate the time averaged moment response amplitudes, and these are plotted against the corresponding input amplitude in Fig. 7. This test was conducted at three different rotor speeds to detect changes in sensitivity due to varying thrust requirements.

Each sensitivity curve displays a cut-in threshold below which no meaningful control moment is generated, then a region of roughly linear growth in generated moment, and finally a plateau where increasing signal amplitude no longer generates increasing aerodynamic moments. The slope of the linear regions appear similar across all three motor speed curves. At higher speeds the required cut-in input amplitude is larger and the ultimate attainable control moment is also elevated. One possible explanation for the cut-in threshold may be the need to overcome static friction at the plasticplastic hinge interface which would be expected to grow with increasing rotor speed and corresponding normal force at the hinge bearing surface. The cause of the saturation in response is unknown, but it was observed that the hard kinematic stops of the hinge were being stressed at very high driving amplitudes. Finally, while the generated moment magnitude was sensitive to both the thrust condition and the driving signal amplitude, the axis direction of the moment remains relatively constant. This is a very desirable feature when integrating this system as a swashplate replacement.

The control sensitivity testing indicates that a maximum control moment of $30 \mathrm{~N} \cdot \mathrm{mm}$ is available from the $38.6 \mathrm{~cm}$ rotor. Comparisons can be made to similar platforms. The tiny Syma S107 is a $40 \mathrm{~g}, 19 \mathrm{~cm}$ diameter toy coaxial helicopter whose pitching tail rotor provides approximately $1.7 \mathrm{~N} \cdot \mathrm{mm}$ of authority to the pilot. The larger Blade 120SR is a $106 \mathrm{~g}, 33 \mathrm{~cm}$ diameter single propeller helicopter whose cyclic control offers $45 \mathrm{~N} \cdot \mathrm{mm}$ of moment. ${ }^{1}$ In contrast, the much larger Ascending Technologies X3D "Hummingbird" quadrotor generates $460 \mathrm{~N} \cdot \mathrm{mm}$ of rolling moment on its $468 \mathrm{~g}$ frame from four $20 \mathrm{~cm}$ diameter rotors mounted on $17 \mathrm{~cm}$ arms, giving it exceptional maneuverability [10]. The $30 \mathrm{~N} \cdot \mathrm{mm}$ of control authority allowed by the present prototype should prove sufficient for simple maneuvers in a range of aircraft sizes, but eccentric payload capacity or aggressive maneuvering capabilities may prove limited.

\footnotetext{
${ }^{1}$ Available pilot moments were estimated by experimentally determining the largest eccentric payload that could be stabilized by one amateur pilot
} 


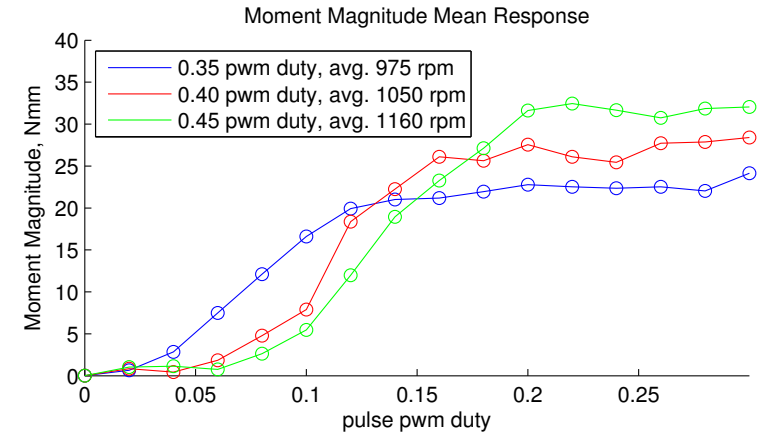

Fig. 7. The net control moment response magnitude increases with increasing amplitude of excitation.

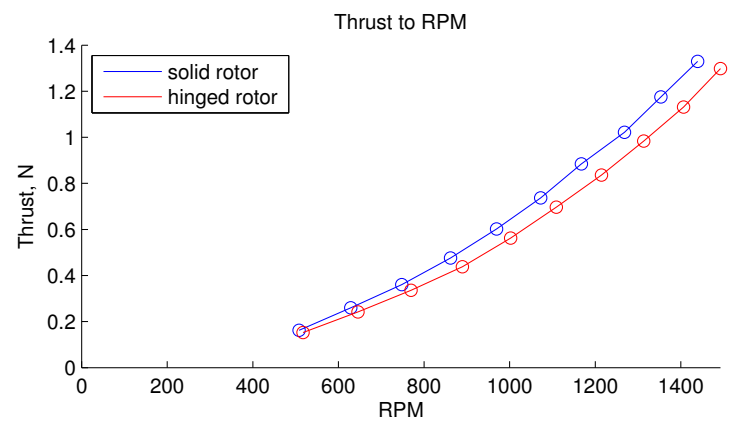

Fig. 8. Thrust curve for similar rigid and hinged propellers.

\section{SteAdy AEROdYNAMIC EFFICIENCY}

Achieving useful flight duration is a challenge in MAVs due to their high power requirements and low capacity for on board energy storage. Flight efficiency for lifting rotor craft is predominately dependent on the lift and drag characteristics of the main rotor. To test whether the new hinged architecture sacrifices thrust efficiency, its performance was compared to a geometrically similar rigid propeller by measuring lift and drag across a range of rotor speeds as in Fig. 8-9. One issue of the new hinged rotor is that in steady state the drag forces bend both blades backwards slightly at their hinges, increasing the angle of attack on one side and decreasing on the other. It is possible to anticipate this degree of deflection due to the small range of operating speeds for a helicopter blade. The rotor blade clamp geometry attempts to compensate for this effect in for optimal angle of attack in the hover condition, however the lift and drag curve comparisons show that rotor performance is distinctly affected.

An important performance metric for lifting rotors is the

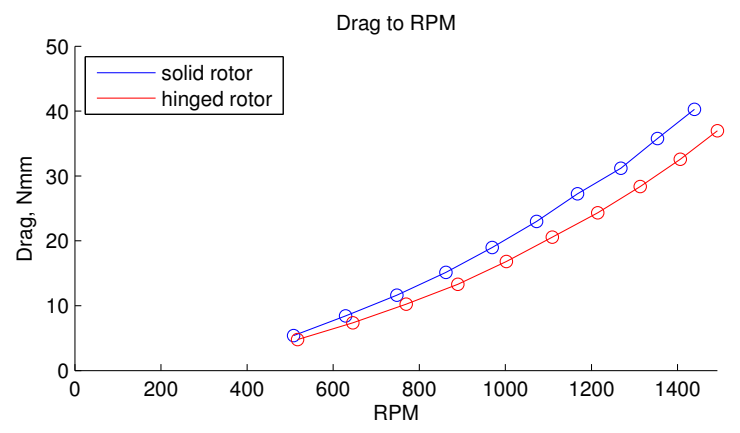

Fig. 9. Drag curve for similar rigid and hinged propellers.

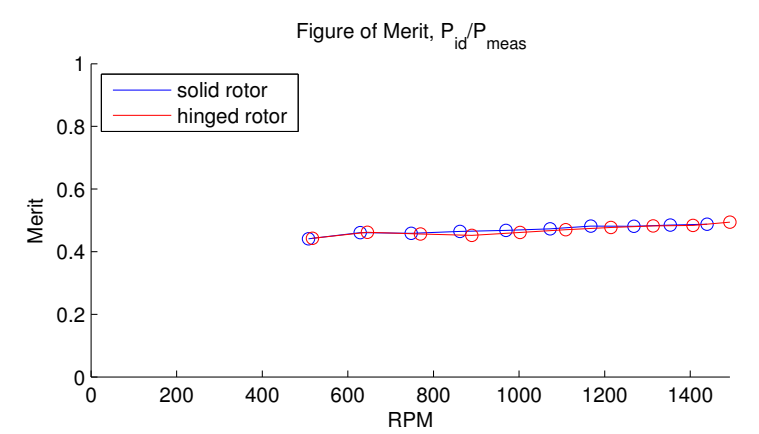

Fig. 10. Figure of merit for geometrically similar hinged and rigid hub propellers.

figure of merit, $F M$, defined as the ideal power divided by the actuator power for a given thrust and incident flow velocity. The actuator mechanical shaft power on the test stand $P_{\text {meas }}$ is given by the product of the applied torque $M$ and the rotor shaft speed $w$. The ideal power $P_{i d}$ is derived from momentum theory by solving for the momentum balance in a control volume around the idealized actuator disk. The ideal power at hover is dependent on the thrust $T$, air density $\rho$, and rotor radius $r$, and this value is used to calculate $F M$ according to equations 1-3 [11].

$$
\begin{gathered}
P_{\text {meas }}=M * w \\
P_{i d}=\frac{T^{\frac{3}{2}}}{\sqrt{2 \rho \pi r^{2}}} \\
F M=\frac{P_{i d}}{P_{\text {meas }}}
\end{gathered}
$$

FM for both the hinged propeller system and the geometrically similar fixed hub propeller are compared in Fig. 10. The hover efficiency of the hinged blade sample is slightly worse than its fixed hub counterpart over most of the operational envelope. This performance analysis suggests that the amount of blade trim adjustment due to drag has been incorrectly estimated in this iteration, and the angle of attack in steady state does not reach its optimum configuration. Future iterations of the zero-deflection angle pitch for the blade clamps will be informed by these results.

\section{Steady CyCliC Response}

The time history of moment response over each rotor revolution was measured in response to a driving oscillatory input voltage amplitude of 0.10 pwm about a mean value of 0.35 pwm duty factor, corresponding to a rotor speed of $931 \mathrm{rpm}$ on a $7.4 \mathrm{~V}$ power supply. Fig. 11 shows the measured vertical axial torque applied to the rotor in black, the moment about the horizontal axis of maximum mean response in blue, and the moment about the horizontal transverse axis in red. In a flight system, a roll response would be directed by aligning the axis of maximum mean response with the roll axis of the airframe. Dotted trend lines show the long term average moment about the excited axis is $14.6 \mathrm{~N} \cdot \mathrm{mm}$, and the average moment about the transverse axis is nearly zero. During these tests a steady once per revolution oscillation in moment was also observed due to dynamic and aerodynamic imbalance in the rotor. This component did not contribute to the mean values of 


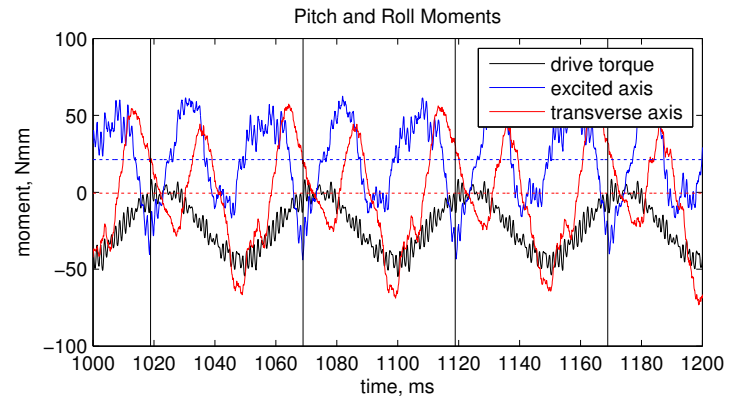

Fig. 11. Cyclic torque and response moment at $1192 \mathrm{rpm}$ with $0.09 \mathrm{pwm}$ duty excitation amplitude. Bars highlight the period of revolution.

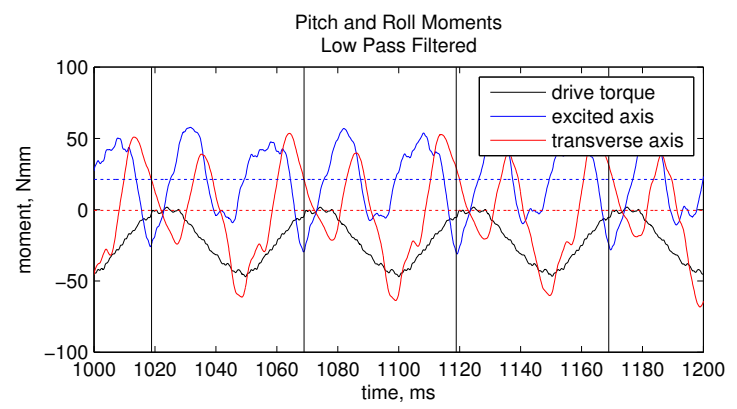

Fig. 12. Low-pass filtered cyclic torque and response moment at $1192 \mathrm{rpm}$ with 0.09 pwm excitation amplitude. Bars highlight the period of revolution.

the control response and was subtracted out to highlight the characteristic twice-per-revolution behavior in these figures. The motor's cogging torque ripple is also represented in both the axial driving torque and the response moments. In Fig. 12 the signals have been post processed with a bidirectional first order Butterworth low pass filter. The filter corner frequency was placed at 12 times the rotor speed in order to attenuate the lowest frequency cogging ripples by $-3 \mathrm{db}$.

The low pass filtered results highlight the expected frequency doubling property of the propeller system, with peaks in the excitation moment corresponding to the passing of each of the two rotor blades. Major and minor peaks indicate that the two propeller blades are not contributing equally to the long term output moment, which suggests the blade motions are not equally sensitive to changing applied torques. Adjusting the hinge-neutral blade pitch on each side may partially alleviate this asymmetry in future iterations.

\section{TRAnsiEnt Response to Step InPUtS}

A practical implementation of the hinged propeller system in a free flight craft requires rapid changes in commanded orienting moment magnitudes and directions. As a result, the transient response to fluctuating torque inputs is just as important as the steady state response sensitivities previously summarized in Fig. 7. The response to step inputs are a good indication of this type of performance. Figs. 13-14 show the raw moment measurements associated with a series of steps in control input at a propeller velocity of $1192 \mathrm{rpm}$. When active, the driving torque traces a sinusoidal history and the orthogonal $\mathrm{x}$ and $\mathrm{y}$ axes reveal twice per revolution oscillations as each blade passes. The background once per revolution oscillation observed even when the modulation is not active is due to an undesirable imbalance in the rotor.

The initial commanded moment is zero with zero input oscillation, at $t=1 \mathrm{~s}$ the input oscillation amplitude steps

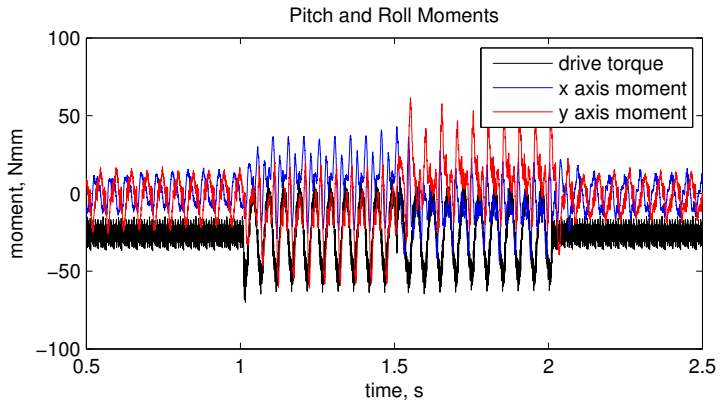

Fig. 13. Three switching events in magnitude and phase.

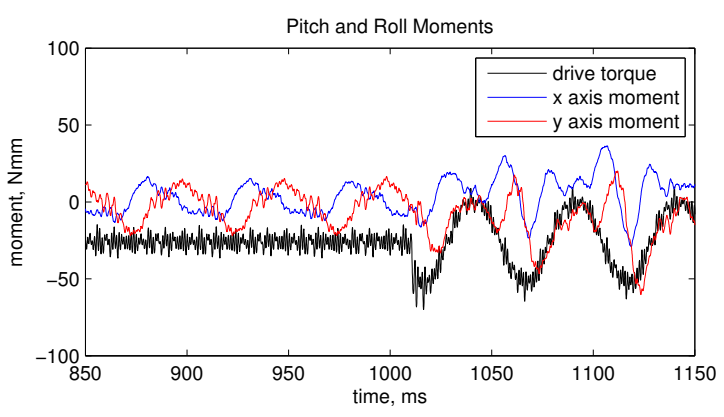

Fig. 14. Highlight of step in modulation amplitude.

to 0.10 pwm duty to command a moment about one axis, at $t=1.5 \mathrm{~s}$ the phase of input oscillation steps by $180^{\circ}$ to command a reversal in orienting moment direction, and at $t=2 \mathrm{~s}$ the commanded moment returns to zero. At each stage the output response waveform settled into the new driven limit cycle within one revolution of the propeller, consistent with a step input rise time of less than $50 \mathrm{~ms}$. This level of performance is comparable to cyclic control helicopter craft which generate body moments through pitch reorientation in a very similar way to the proposed hinged propeller system. Quadrotor systems, on the other hand, must generate variations in body moment by changing the thrust of each propeller. This ramp in thrust has been characterized for the popular Ascending Technologies X3D "Hummingbird" quadrotor as a first order linear process with a rise time of $44 \mathrm{~ms}$ for rising and $88 \mathrm{~ms}$ for falling inputs [10]. Others have attempted to improve on this benchmark by augmenting the quadrotor with collective pitch actuators and have achieved similar rise and fall times of $40 \mathrm{~ms}$ [3]. In this light, the capacity for arbitrary moment modifications within a single propeller revolution makes the hinged rotor concept competitive with widely adopted MAV technologies.

\section{Flight System Design and Testing}

A complete MAV system was developed to demonstrate how this novel rotor can be successfully incorporated into an airframe, shown in Fig. 15. The MAV adopts a coaxial rotor configuration with a standard fixed pitch propeller on the bottom and the underactuated hinged rotor on top. The vehicle has no passive stability due to the lack of the traditional flybar mechanism. No servo-driven swashplate, auxiliary pusher propellers, or thrust vectoring techniques are employed - the stability and controllability of the vehicle are due exclusively to the dynamic response of the top propeller to the electronic modulation of the main rotor torque. The final aircraft supports a $30 \mathrm{~cm}$ diameter bottom rotor and a 


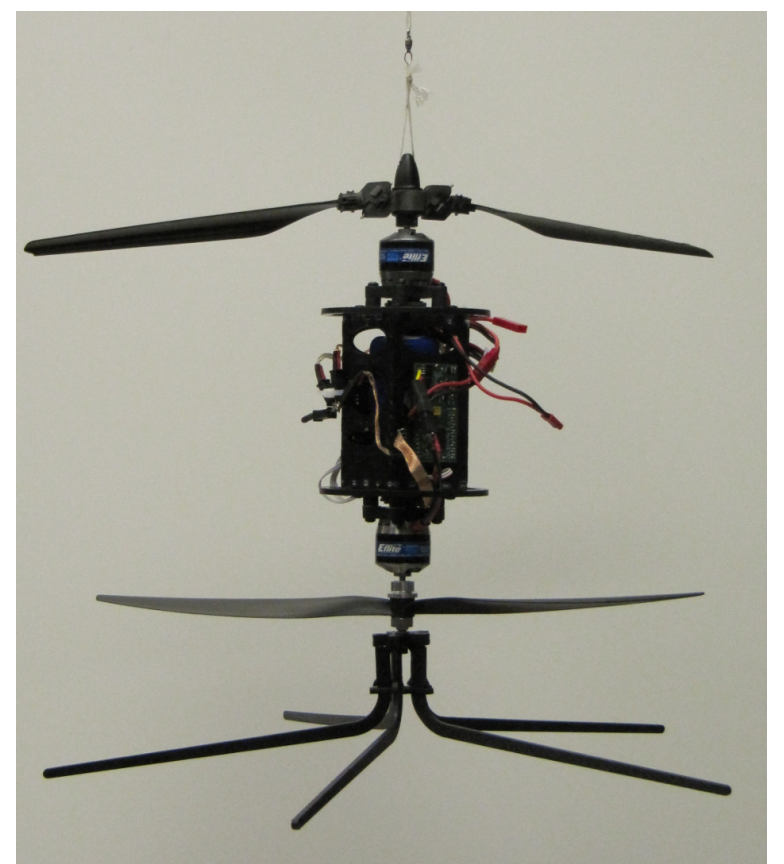

Fig. 15. MAV incorporating a top hinged rotor and bottom fixed rotor. TABLE II

Component Masses of the CoAXial MAV

\begin{tabular}{|r|r|}
\hline component & mass \\
\hline \hline hinged propeller and mandrel & $21.0 \mathrm{~g}$ \\
\hline fixed propeller and mandrel & $16.6 \mathrm{~g}$ \\
\hline brushless motors & $2 \times 54.8 \mathrm{~g}$ \\
\hline motor controllers & $2 \times 10.5 \mathrm{~g}$ \\
\hline inertial measurement unit (IMU) & $2.5 \mathrm{~g}$ \\
\hline radio and attitude controller & $12.3 \mathrm{~g}$ \\
\hline lithium polymer battery & $70.4 \mathrm{~g}$ \\
\hline landing gear & $20.8 \mathrm{~g}$ \\
\hline airframe and misc. hardware & $84 . \mathrm{g}$ \\
\hline total & $358 . \mathrm{g}$ \\
\hline
\end{tabular}

$38.6 \mathrm{~cm}$ top rotor separated by $28 \mathrm{~cm}$ vertical distance, and it has a gross weight of $358 \mathrm{~g}$.

The control implementation illustrated in Fig. 16 segments the system into an onboard inertial measurement unit (IMU), a central attitude controller, and two motor controllers. The IMU provides yaw, pitch, and roll attitude estimates for the airframe. The attitude controller calculates yaw, pitch, and roll corrective moments based on these estimates through a linear proportional-derivative controller. These corrections map to a mean voltage, modulation amplitude, and modulation phase offset which are sent to the motor controllers. Yaw correction is applied through a difference between the applied mean voltages for the top and bottom counter-rotating rotors, a traditional technique in coaxial MAV. Pitch and roll corrections are vectorially added to generate a magnitude and angle in the rotor plane, and these are conveyed to the top motor as modulation amplitude and phase offsets. Finally, the motor controllers drive the normal electrical commutation of the brushless motor, but additionally add a rotor-angle locked sinusoidal signal to the mean command voltage in order to generate the sinusoidally pulsing torque.

The MAV has proved capable of hovering stably in indoor tests, needing pilot intervention only to maintain altitude and correct slow lateral drift. As demonstrated in the companion

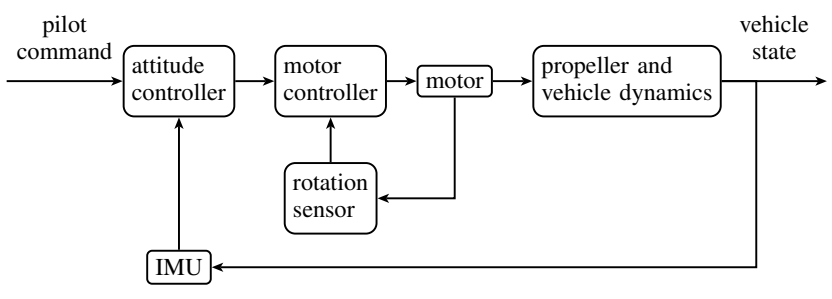

Fig. 16. Block diagram for the MAV on board control system.

video, the pilot can maneuver the craft by sending desired pitch and roll moments which augment the normal outputs of the stability controller.

\section{CONClusions}

We have shown that a hinged, underactuated rotor can mimic the behavior of traditional cyclic control systems in small MAVs without requiring either additional servomotor actuators or complex linkage systems. Both the magnitude and response time of the resulting control moments are sufficient for stabilizing and maneuvering a small, $358 \mathrm{~g}$ coaxial MAV. In future work, we wish to develop a technique for determining optimal geometric design parameters for power-efficient operation given application constraints on required moments, thrust, and rotor size. This will allow us to evaluate the system-level power efficiency of this technology verses other control strategies while taking into account the associated actuator and structure material weights. The ultimate aim of this technology is to achieve reductions in system complexity and actuator count that may enable future small, simple, and low cost micro air vehicles.

\section{ACKNOWLEDGEMENT}

The authors wish to thank Matt Piccoli for developing the custom motor controllers used in these experiments.

\section{REFERENCES}

[1] A. Kushleyev, et al., "Towards a swarm of agile micro quadrotors," in Robotics: Science and Systems, 2012.

[2] A. Briod, et al., "The AirBurr: a flying robot that can exploit collisions," in 2012 ICME International Conference on Complex Medical Engineering (CME), July 2012, pp. 569-574.

[3] M. Cutler, et al., "Comparison of fixed and variable pitch actuators for agile quadrotors," in AIAA Guidance, Navigation, and Control Conference (GNC), 2011.

[4] S. George and P. Samuel, "On the design and development of a coaxial nano rotorcraft," in 50th AIAA Aerospace Sciences Meeting. American Inst. of Aeronautics and Astronautics, Jan. 2012.

[5] D. Schafroth, et al., "From the test benches to the first prototype of the muFly micro helicopter," Journal of Intelligent and Robotic Systems, vol. 54, no. 1-3, pp. 245-260, Mar. 2009.

[6] M. Keennon, et al., "Development of the nano hummingbird: A tailless flapping wing micro air vehicle," in 50th AIAA Aerospace Sciences Meeting. American Inst. of Aeronautics and Astronautics, Jan. 2012.

[7] C. Richter and H. Lipson, "Untethered hovering flapping flight of a 3D-Printed mechanical insect," Artificial Life, vol. 17, no. 2, pp. 73-86, Mar. 2011.

[8] Y. Fujihira, et al., "Propeller blade tip path plane inclining device," U.S. Patent 5259729 , Nov., 1993.

[9] S. K. Kim and D. M. Tilbury, "Mathematical modeling and experimental identification of an unmanned helicopter robot with flybar dynamics," J. of Robotic Syst., vol. 21, no. 3, pp. 95-116, Mar. 2004.

[10] S. Lupashin, et al., "A simple learning strategy for high-speed quadrocopter multi-flips," in 2010 IEEE International Conference on Robotics and Automation (ICRA), May 2010, pp. 1642-1648.

[11] G. J. Leishman, Principles of Helicopter Aerodynamics, 2nd ed. Cambridge University Press, Apr. 2006. 